\title{
Pubertal maturation and sex effects on the default-mode network connectivity implicated in mood dysregulation
}

Monique Ernst', Brenda Benson', Eric Artiges ${ }^{2,3,4,5,6}$, Adam X. Gorka', Herve Lemaitre $\mathbb{C}^{2,3}$, Tiffany Lago', Ruben Miranda ${ }^{2}$, Tobias Banaschewski ${ }^{7}$, Arun L. W. Bokde ${ }^{8}$, Uli Bromberg $^{9}$, Rüdiger Brühll ${ }^{10}$, Christian Büchel $^{9}$, Anna Cattrell ${ }^{11}$, Patricia Conrod ${ }^{12,13}$, Sylvane Desrivières (10 ${ }^{11}$, Tahmine Fadai ${ }^{9}$, Herta Flor ${ }^{7,14}$, Antoine Grigis ${ }^{15}$, Juergen Gallinat ${ }^{16}$, Hugh Garavan ${ }^{17}$, Penny Gowland ${ }^{18}$, Yvonne Grimmer ${ }^{7}$, Andreas Heinz ${ }^{19}$, Viola Kappel ${ }^{20}$, Frauke Nees ${ }^{7,14}$, Dimitri Papadopoulos-Orfanos $\mathbb{D}^{15}$, Jani Penttilä ${ }^{21}$, Luise Poustka,22, Michael N. Smolka $\mathbb{E}^{23}$, Argyris Stringaris ${ }^{1,24}$, Maren Struve ${ }^{25}$, Betteke M. van Noort ${ }^{20}$, Henrik Walter $\mathbb{1}^{19}{ }^{19}$, Robert Whelan ${ }^{25}$, Gunter Schumann ${ }^{11}$, Christian Grillon", Marie-Laure Paillère Martinot ${ }^{2,4,26,27}$ and Jean-Luc Martinot ${ }^{2,3,4,5}$, for the IMAGEN Consortium

\begin{abstract}
This study examines the effects of puberty and sex on the intrinsic functional connectivity (iFC) of brain networks, with a focus on the default-mode network (DMN). Consistently implicated in depressive disorders, the DMN's function may interact with puberty and sex in the development of these disorders, whose onsets peak in adolescence, and which show strong sex disproportionality (females $>$ males). The main question concerns how the DMN evolves with puberty as a function of sex. These effects are expected to involve within- and between-network iFC, particularly, the salience and the central-executive networks, consistent with the Triple-Network Model. Resting-state scans of an adolescent community sample ( $n=304$, male/female: 157/147; mean/std age: 14.6/0.41 years), from the IMAGEN database, were analyzed using the AFNI software suite and a data reduction strategy for the effects of puberty and sex. Three midline regions (medial prefrontal, pregenual anterior cingulate, and posterior cingulate), within the DMN and consistently implicated in mood disorders, were selected as seeds. Within- and between-network clusters of the DMN iFC changed with pubertal maturation differently in boys and girls (puberty-X-sex). Specifically, pubertal maturation predicted weaker iFC in girls and stronger iFC in boys. Finally, iFC was stronger in boys than girls independently of puberty. Brain-behavior associations indicated that lower connectivity of the anterior cingulate seed predicted higher internalizing symptoms at 2-year follow-up. In conclusion, weaker iFC of the anterior DMN may signal disconnections among circuits supporting mood regulation, conferring risk for internalizing disorders.
\end{abstract}

\section{Introduction}

Puberty and sex critically influence brain maturation in adolescence (for review, see ref. ${ }^{1,2}$ ). The pubertal rise in

\footnotetext{
Correspondence: Monique Ernst (ernstm@mail.nih.gov)

${ }^{1} \mathrm{NIMH} / \mathrm{NIH}$, Bethesda, MD, USA

${ }^{2}$ INSERM, UMR 1000, Research unit "Neuroimaging and Psychiatry", DIGITEO

Labs, University Paris-Saclay, and University Paris Descartes, Gif sur Yvette,

France

Full list of author information is available at the end of the article.
}

sex steroids is thought to further refine the organizational sex differences that are established early in life (for review, see ref. ${ }^{3,4}$. These puberty-related effects are expected to contribute to brain development and to promote sex differences in neural circuits. Consistent with this notion, puberty-related changes in brain functional organization would be predicted to reveal different trajectory patterns between girls and boys. The influence of puberty on brain function may also have a role in the emergence of

\section{(c) The Author(s) 2019}

(c) (i) Open Access This article is licensed under a Creative Commons Attribution 4.0 International License, which permits use, sharing, adaptation, distribution and reproduction cc) in any medium or format, as long as you give appropriate credit to the original author(s) and the source, provide a link to the Creative Commons license, and indicate if changes were made. The images or other third party material in this article are included in the article's Creative Commons license, unless indicated otherwise in a credit line to the material. If material is not included in the article's Creative Commons license and your intended use is not permitted by statutory regulation or exceeds the permitted use, you will need to obtain permission directly from the copyright holder. To view a copy of this license, visit http://creativecommons.org/licenses/by/4.0/. 
psychiatric disorders, for two reasons. First, incidence rates of psychiatric disorders peak in adolescence ${ }^{5}$, and, second, they exhibit striking sex differences ${ }^{6}$, such as female preponderance for depressive and anxiety disorders. In addition, a specific role of sex steroids, such as high levels of dehydroepi-androsterone in children, has been associated with mental health problems (e.g., ref. ${ }^{7}$ ).

In contrast to the abundant neurodevelopmental research probing neural changes with age and sex (e.g., ref. ${ }^{8-18}$ ), neuroimaging studies that query the effects of puberty on neural development, are relatively sparse ${ }^{19-26}$ (for review, see ref. ${ }^{27}$ ). A primary focus of these neurodevelopmental studies have targeted structural measures of the brain (e.g., ref. ${ }^{28-31}$ and task-based functional magnetic resonance imaging (fMRI ${ }^{20-24}$ ). Relatively few resting-state fMRI studies have investigated the effects of age and sex in adolescence. Across the relative sparsity of these studies, many different methods and targets have been used, which make the identification of consistent patterns too soon to draw. Some studies focus on specific structures. For example, Alarcon et al. ${ }^{23}$ examined iFC of subregions of the amygdala (seeds) in 122 healthy youths (10-16 years). Their findings show opposite age-related changes in connectivity in girls vs. boys, but these changes are not always in the same direction depending on the amygdala subregions. Others have conducted studies of whole-brain organization, using methods such as graph theory (for review ref. ${ }^{32}$ ). For example, Satterthwaite et al. examined sex differences in 9-22-year-old youths ${ }^{33}$. They reported sex differences in the organization of wholebrain connectivity (i.e., greater between-networks connectivity in boys, and greater within-networks connectivity in girls). This type of data underscore the presence of sex differences in brain connectivity in youths, but does not speak specifically to the direction of sex differences in specific networks. Unfortunately, an analogous situation characterizes this type of research in adults (e.g., $\left.{ }^{34-36}\right)$. Of note, a recent study using a large adult sample from the human connectome $(n=820,336$ females, 22-37 yo) identified the default-mode network (DMN) as being the best predictor of sex status, particularly for couplings involving the fusiform gyrus and ventromedial prefrontal corte ${ }^{37}$. The direction of effects was not detailed. Taken together, this brief survey of the literature does not permit to integrate existing findings into specific hypotheses that could guide the present work. Finally, to our knowledge, no studies have yet investigated puberty-related changes in resting-state functional connectivity (referred to as "intrinsic Functional Connectivity" or iFC), particularly with respect to the DMN, which is shown to have a central role in the development of psychopathology 38 , and seems to be highly sensitive to sex status ${ }^{37}$. Of note, one reason for the sparsity of research on puberty stems from the difficulty of dissociating the effects of age from puberty.

The present study takes advantage of a large community cohort of adolescents, all $\sim 14$-year old ${ }^{39}$, to examine how puberty and sex influence the iFC of three specific nodes. These three nodes have been selected for two reasons. First, their structural parameters have been associated with adolescent mood dysregulation ${ }^{40,41}$. Second, they belong to the $\mathrm{DMN}^{42,43}$. As mentioned above, the DMN has been consistently implicated in internalizing disorders (e.g., ref. ${ }^{44-48}$ and it comprises midline cortical regions that systematically emerge in studies of mood disorders (see reviews and meta-analyses ${ }^{45,49-54}$ ). Therefore, understanding the effects of puberty and sex on the development of the DMN function might shed light on the neural mechanisms conferring vulnerability to mood dysregulation in adolescence.

Highly relevant to this question is the Triple-Network Model $^{55}$. This model proposes that dysfunction or imbalance among three core canonical networks of resting-state fMRI might contribute to a number of psychiatric disorders. These networks consist of the DMN, the central-executive network and the salience network (SN). The DMN serves self-referential-related functions and comprises regions in the anterior medial prefrontal cortex (PFC), posterior cingulate cortex, middle temporal cortex, and hippocampus ${ }^{47,48}$. The central-executive network supports working memory, decision-making, and cognitive control. This latter network is particularly important for the regulation of emotion processing, which itself depends largely on subcortical regions (e.g., amygdala). The central-executive network encompasses the dorsolateral PFC and dorsomedial PFC ${ }^{56-58}$. Finally, the SN supports the integration of internal and external stimuli into emotional and behavioral responses. The core nodes of the $\mathrm{SN}$ include the insula and dorsal anterior cingulate cortex ${ }^{59}$. The framework of the Triple-Network Model is used as a heuristic tool in the present work. This approach emulates the widely use of neural systems models to explain typical adolescent behaviors, such as increased risk-taking, emotional lability, or social transformation $^{60-64}$.

The present work focuses on how puberty and sex affect the DMN iFC, including couplings within and between networks, especially the salience and central-executive networks of the Triple-Network Model. We hypothesize sex differences in the pubertal maturation effects on the brain's iFC. We also anticipate sex differences that are independent of pubertal maturation ${ }^{1}$. However, we do not predict pubertal maturation to influence brain connectivity similarly in males and females, because puberty is by essence sexually dimorphic. Specific directional hypotheses are difficult to predict, based on the lack of 
prior work of typical changes of resting-state networks with puberty. Finally, exploiting the 2-year behavioral follow-up (16 yo) of this cohort, exploratory brain-behavior analyses are expected to reveal associations between the DMN iFC of the 14-year old and behavioral dimensions of internalizing, externalizing, or social problems when these adolescents reach 16 years of age.

\section{Participants and methods \\ Participants}

The IMAGEN consortium recruited over 2000 youths. Only five sites opted to collect resting-state scans. IMAGEN data are available from a dedicated database: https:// imagen2.cea.fr. Adolescents $(n=381)$ from the IMAGEN sample $^{39}$ underwent fMRI scanning in a resting state. A sample size of $n=381$ subjects was expected to provide sufficient power to detect reliable interaction effects of puberty by sex on iFC measures. Indeed, previous studies have reported significant clinical group effects on measures of intrinsic connectivity in pediatric samples smaller than $n$ $=60$ (e.g., ref. ${ }^{65-67}$ ). Recruitment and assessment procedures, and exclusion and inclusion criteria are described elsewhere in detail ${ }^{39}$. In brief, participants and their parents were recruited via middle-schools in five European sites. Inclusion criterion was age between 13 and 15 years. Exclusion criteria were birth weight $<800 \mathrm{~g}$, severe medical conditions, bipolar disorder, treatment for schizophrenia, and major neuro-developmental disorders. All participants were assessed for intelligence quotient (IQ) using the Wechsler Abbreviated Scale of Intelligence ${ }^{68}$.

Written informed assent and consent were obtained, respectively, from all adolescents and their parents in accordance with the ethics committees of the participating institutions $^{39}$ and the Declaration of Helsinki. Seventyseven participants were excluded from the analysis owing to excessive head motion (i.e., $>30 \%$ of acquired Repetition Time (TRs) with a frame-to-frame Euclidean norm motion derivative $>0.25 \mathrm{~mm} ; n=72,53$ boys and 19 girls), poor spatial normalization by visual inspection $(n=2)$, or corrupted data $(n=1)$. Two participants lacked pubertal scores. The excluded group $(n=77)$, compared to included participants $(n=304)$, had more boys $(T=3.58, p<$ 0.001 ), but was similar in age, puberty status, and IQ (all $p$ 's $>0.1$ ). Characteristics of the sample are presented in Table 1. Of note, participants overlapped slightly with those of our previous structural studies (i.e., $n=21$ in common with ${ }^{40}, n=31$ in common with Vulser et al. ${ }^{41}$, and $n=5$ in common to all three samples).

Behavioral data were collected again in this sample 2 years later, at age 16 years. The attrition rate was $17 \%$ (53 subjects were not tested at follow-up), leaving a sample of 251 16-yo adolescents.

\section{Behavioral assessments}

Every participant completed a psychiatric assessment via the Development and Well-Being Assessment (DAWBA, www.dawba.com). The DAWBA is a selfadministered questionnaire consisting of open- and closeended questions completed by the participants and their parents that generates computerized probability levels of meeting DSM-IV and ICD-10 diagnoses, called "DAWBA bands" that are subsequently validated by experienced clinicians $^{69}$. As part of the DAWBA, participants also completed a self-report inventory behavioral screening questionnaire, the Strengths and Difficulties questionnaire

Table 1 Demographic information

\begin{tabular}{|c|c|c|c|c|c|c|}
\hline \multirow{3}{*}{ Demographics no. of participants } & \multicolumn{2}{|c|}{ All participants } & \multicolumn{2}{|c|}{ Male participants } & \multicolumn{2}{|c|}{ Female participants } \\
\hline & \multicolumn{2}{|l|}{$N=304$} & \multirow{2}{*}{$\begin{array}{l}N=157 \\
\text { Mean }\end{array}$} & \multirow{2}{*}{$\begin{array}{l}(51.6 \%) \\
( \pm 1 \mathrm{SD})\end{array}$} & \multirow{2}{*}{$\begin{array}{l}N=147 \\
\text { Mean }\end{array}$} & \multirow{2}{*}{$\begin{array}{l}\text { (48.4\%) } \\
\text { ( } \pm 1 \mathrm{SD})\end{array}$} \\
\hline & Mean & $( \pm 1 \mathrm{SD})$ & & & & \\
\hline Age (days) & 5279 & \pm 151 & 5265 & \pm 149 & 5294 & \pm 152 \\
\hline Puberty (PDS) & 2.86 & \pm 0.57 & 2.56 & \pm 0.55 & 3.18 & \pm 0.38 \\
\hline $1 Q^{b}$ & 107 & \pm 12 & 107 & \pm 12 & 108 & \pm 12 \\
\hline Scanner site 1 (Dublin) & \multicolumn{2}{|l|}{$N=26$} & \multicolumn{2}{|l|}{$N=8$} & \multicolumn{2}{|l|}{$N=18$} \\
\hline Scanner site 2 (London) & \multicolumn{2}{|l|}{$N=42$} & \multicolumn{2}{|l|}{$N=42$} & \multicolumn{2}{|l|}{$N=0$} \\
\hline Scanner site 3 (Dresden) & \multicolumn{2}{|l|}{$N=116$} & \multicolumn{2}{|l|}{$N=57$} & \multicolumn{2}{|l|}{$N=59$} \\
\hline Scanner site 4 (Mannheim) & \multicolumn{2}{|l|}{$N=54$} & \multicolumn{2}{|l|}{$N=19$} & \multicolumn{2}{|l|}{$N=35$} \\
\hline Scanner site 5 (Paris) & \multicolumn{2}{|l|}{$N=66$} & \multicolumn{2}{|l|}{$N=31$} & \multicolumn{2}{|l|}{$N=35$} \\
\hline
\end{tabular}

PDS Pubertal Development Scale, IQ intelligent quotient. Demographic information for the whole sample, male participants, and female participants a (females $>$ males, $p<0.05$ )

${ }^{b} \mathrm{IQ}$ measured using the Wechsler Abbreviated Scale of Intelligence (WASI) 
$\left(\mathrm{SDQ}^{70}\right)$, which gives a measure of severity of problems within internalizing, externalizing, and social behavioral domains. Although the resting-state fMRI scanning only occurred at age 14 years, the behavioral assessments were repeated 2 years later, at age 16 years.

\section{Pubertal status}

Pubertal status was assessed via self-report using the Pubertal Development Scale (PDS) ${ }^{71}$. Previous research has demonstrated that the PDS exhibits good internal consistency (median $\alpha=0.77$ ) and is highly correlated with physician ratings (Pearson's $R=0.61)^{71,72}$.

\section{BOLD fMRI data acquisition, preprocessing, and analysis}

MRI data were acquired at five sites using $3 \mathrm{~T}$ scanners: Phillips (Dublin), General Electrics (London), and Siemens (Paris, Dresden, Mannheim). BOLD fMRI signal was acquired across 40 interleaved slices using the following parameters: $\mathrm{TR}=2,200 \mathrm{~ms}$; $\mathrm{TE}=30 \mathrm{~ms}$; flip angle $=75^{\circ}$; acquisition matrix $=64 \times 64 \times 40$ with $2.4 \mathrm{~mm}$ slice thickness and $1 \mathrm{~mm}$ slice gap yielding an acquisition resolution $3.4 \mathrm{~mm}$ isotropic; 187 volumes collected over 6.5 minutes. High-resolution anatomical images were obtained using parameters based on the ADNI protocol, yielding a final voxel size of $1.1 \times 1.1 \times 1.1 \mathrm{~mm}$.

Preprocessing and analyses of BOLD fMRI data were conducted using $\mathrm{AFNI}^{73}$. FreeSurfer version $5.3^{74}$ was employed to segment the T1-weighted anatomical images. The ifirst four volumes of the functional run were discarded to allow for steady-state equilibrium. Functional volumes were slice-time corrected, aligned, and coregistered to the participants' corresponding anatomical image. Functional volumes were then normalized to the Colin 27 Average Brain standardized template using 3dQwarp, which is a nonlinear transformation, and spatially smoothed with a $6 \mathrm{~mm}$ Full-Width Half-Maximum (FWHM) Gaussian kernel. All coordinates are reported in the Talairach and Tournoux system ${ }^{75}$.

Acknowledging the debate as to whether global signal should be regressed out of resting-state data sets, we decided not to adopt this strategy. This decision was based on Saad et al. ${ }^{76}$ who suggest that global signal regression can introduce spurious correlations into resting-state data sets, and advise against its use in preprocessing. In addition, we employed several strategies to minimize motion- and physiological-related variance, thus mitigating the need to apply additional measures, like global signal regression, to minimize variance related to motion and cardiac/respiratory processes (Power et al. ${ }^{77}$ ).

The following nuisance signals were regressed from the functional volumes: (1) six head motion parameters and their derivatives, (2) average time-series extracted from the ventricles, (3) time-series from local white matter within a $25-\mathrm{mm}$ radius sphere surrounding each voxel using the
ANATICOR approach ${ }^{78}$, and (4) individual regressors corresponding with a frame-to-frame Euclidean norm motion derivative $\geq 0.25 \mathrm{~mm}$, or volumes where $\geq 10 \%$ of voxels were determined to be outliers. This strategy followed the recommendations of Power et al. ${ }^{79}$.

Three cortical regions of interest (ROIs) from the $\mathrm{DMN}^{80}$ were selected as seeds. These three seeds were retained because of their previously demonstrated association with subthreshold elevated ${ }^{40}$, as well as depres$\operatorname{sed}^{41}$ mood symptoms in two independent subsets of the community cohort of 14 yo from the IMAGEN consortium (https://imagen.cea.fr ${ }^{39}$ ). MNI coordinates were all converted to Talairach coordinates using the Yale mni2tal GUI ("MNI- Yale University" 2017). Specifically, cubic ROIs $(3 \mathrm{~mm} \times 3 \mathrm{~mm} \times 3 \mathrm{~mm})$ were created within the left pregenual ACC (lpgACC; Talairach: $x=-12, y=$ 36, $z=12$ ), left medial PFC (lmPFC; Talairach: $x=-2$, $y=45, z=16$ ), and the left PCC (Talairach: $x=-1, y=$ $\left.-47, z=34^{81}\right)$. The left side was selected because the regions identified in previous studies were on the left side. Generically, these cortical regions have been implicated in the processing of salience and emotion encoding, particularly in the context of social and self-referential information $(\mathrm{mPFC})^{82}$, visual stimuli $(\mathrm{PCC})^{83}$, and autonomic visceral signals $(\mathrm{pgACC})^{84}$.

Subsequently, average time-series from seed ROIs were extracted from the residualized functional images and were used to calculate Pearson correlations between the time-series from the seed ROIs and every voxel in the brain. Resulting statistical images were Fishertransformed for group analyses.

ANCOVA models (3dMVM) were used to determine the interaction of puberty-X-sex and the main effects of each factor ${ }^{85}$. All group-level ANCOVA analyses statistically controlled for the effects of scanner site (Dublin, Dresden, London, Mannheim, and Paris), as well as age. Group-level analyses were limited to all gray matter regions as determined by the parcellation of the Colin 27 Average Brain (i.e., CA_N27_ML atlas). Statistical thresholding was calculated using 3dClustSim's Monte Carlo simulation via updated versions of 3dFWHMx and 3dClustSim to address the concerns of inflated false positive rates identified by Eklund ${ }^{86}$. These updates incorporate a mixed autocorrelation function that better models non-Gaussian noise structure ${ }^{87}$. The resulting maps were thresholded to $p<0.005$ two-tailed, $k=37$, which represents a global cluster correction at $p<0.05$. Finally, to examine more stringently potential effects of group motion, we conducted an additional analysis including the additional covariate of individual average motion per TR. This analysis is presented in supplemental material (Table S1).

Average iFC values from clusters exhibiting a puberty$\mathrm{X}$-sex interaction, or main effects of sex or puberty, were 
extracted using 3dmaskave. Post hoc comparisons were conducted within SPSS v24 and interactions were probed using the PROCESS v $216^{88}$ macro by calculating the beta value for the relationship between puberty and iFC for male and female participants, while controlling for age and scanner site. Levene's test for equality of variances, and measures of skew and kurtosis are reported in Supplemental Table S2. Importantly, all clusters exhibiting an interaction between puberty and sex are normally distributed. Furthermore, although variance is not equal between groups for all identified clusters, multiple regression analyses, which do not entail equality of variance assumptions, demonstrate that identified interactions remain significant within a multiple regression framework (Supplemental Tables S3-S4).

\section{Brain-behavior analyses based on factorial approach}

To reduce the number of variables, two sets of principal components using SAS-9.4 program were extracted from the neural data at baseline (14 years old), and the behavioral data collected at 2-year follow-up (16 years old). All the iFC clusters significantly modulated by puberty and/or sex were entered into one factor analysis, and 15 items of the SDQ into a separate factor analysis. These factor analyses used ones as prior communality estimates. The principal axis method was employed to extract the components, and this was followed by a varimax (orthogonal) rotation.

Only two DAWBA bands were retained for this analysis, generalized anxiety disorder and depression, based on our main interest in these frequently comorbid diagnoses ${ }^{89,90}$. The SDQ has 33 items. A total of 16 items were removed a priori, because of no interest. These no-interest items included nine positive items (e.g., "considerate"), seven general items (e.g., "impact at home"). Two additional items ("clingy", "unhappy") were removed because they loaded on multiple factors. Accordingly, the final factor analysis was conducted on 15 items of the SDQ and the two DAWBA bands that probe generalized anxiety disorder and depression. These items and corresponding factor loadings are presented in Table 2. A three-factor resolution was found to be optimal. Combined, factors 1 , 2 , and 3 accounted for $30 \%, 29 \%$, and $21 \%$ of the total variance respectively. When interpreting the rotated factor pattern, an item was said to load on a given factor if the factor loading was 0.40 or greater for that factor and $<$ 0.40 for the others. Using these criteria, six items were found to load on the first factor, which was subsequently labeled "internalizing symptoms". Five items were found to load on the second factor, which was subsequently labeled "externalizing symptoms". Finally, three items were found to load on the third factor, which was subsequently labeled "social problems".
Table 2 Principal component analysis of behavioral variables from the strengths and difficulties questionnaire $\left(\mathrm{SDQ}^{59}\right)$, and the development and well-being assessment (DAWBA, www.dawba.com) at follow-up (16 yo) and variance explained by each factor

\begin{tabular}{|c|c|c|c|}
\hline \multicolumn{4}{|c|}{ Variance explained by each factor } \\
\hline Internalizing & externalizing & So & problems \\
\hline \multirow[t]{3}{*}{2.9905752} & 2.9182511 & \multicolumn{2}{|c|}{2.1157313} \\
\hline & Factor-1 & Factor-2 & Factor-3 \\
\hline & Internalizing & Externalizin & Social \\
\hline Somatic & $52^{\mathrm{a}}$ & 22 & 10 \\
\hline Worries & $63^{\mathrm{a}}$ & 8 & 16 \\
\hline Afraid & $67^{\mathrm{a}}$ & 13 & 9 \\
\hline Impact & $72^{\mathrm{a}}$ & 20 & 14 \\
\hline Depression $^{\mathrm{b}}$ & $69^{a}$ & 18 & -14 \\
\hline Generalized anxiety ${ }^{b}$ & $81^{\mathrm{a}}$ & 13 & 8 \\
\hline Fidgety & 16 & $75^{\mathrm{a}}$ & -2 \\
\hline Restless & 15 & $78^{\mathrm{a}}$ & -1 \\
\hline Distractible & 13 & $67^{\mathrm{a}}$ & -10 \\
\hline Conduct problems & 24 & $50^{\mathrm{a}}$ & 16 \\
\hline Hyperactive & 19 & $94^{\mathrm{a}}$ & -6 \\
\hline Solitary & -4 & -3 & $79^{a}$ \\
\hline Relates better to adults & 17 & -3 & $70^{\mathrm{a}}$ \\
\hline Peer problems & 20 & 2 & $93^{\mathrm{a}}$ \\
\hline
\end{tabular}

Printed values are multiplied by 100 and rounded to the nearest integer. Values $>0.4$ are flagged by an ' $a$ '

The SDQ gives a measure of severity of problems within internalizing, externalizing, and social behavioral domains

${ }^{b}$ DAWBA band: the DAWBA bands represent the probability levels of meeting DSM-IV and ICD-10 diagnoses

A similar approach was adopted to reduce the number of neural variables (iFC clusters). As reported below in the results, 17 clusters were of interest. Four clusters were removed because they loaded on more than one factor. The final 13 clusters and corresponding factor loadings are presented in Table 3. The optimal solution was a three-factor model. Factors 1, 2, and 3 accounted for $49 \%$, $30 \%$, and $27 \%$ of the total variance, respectively. The interpretation of the rotated factor pattern, used the same threshold as above, i.e., 0.40. Using these criteria, eight clusters were found to load on the first factor. These eight clusters were iFC clusters of the IPCC seed $(n=5$ clusters) and of the IMPFC seed ( $n=3$ cluster), all of which being modulated by Puberty-X-Sex. Therefore, factor-1 was labeled PCC/mPFC-pubXsex. Four clusters loaded on the second factor. All these clusters belonged to the IPCCseed iFC, and were sensitive to Sex. Factor-2 was labeled 
Table 3 Principal component analysis of the intrinsic functional connectivity of the three seeds influenced by sex and puberty-X sex(14 yo), and variance explained by each factor

\begin{tabular}{lll}
\hline \multicolumn{2}{l}{ Variance explained by each factor } \\
\hline PCC/mPFC-pubXsex & PCC-sex & ACC-sex \\
\hline 4.8571200 & 2.9548272 & 2.7282190 \\
\hline
\end{tabular}

\begin{tabular}{|c|c|c|}
\hline \multicolumn{3}{|c|}{ Rotated factor pattern } \\
\hline Factor-1 & Factor-2 & Factor-3 \\
\hline PCC/mPFC-pubXsex & PCC-sex & ACC-sex \\
\hline
\end{tabular}

IpgACC-seed sex

$\begin{array}{llll}\text { L_dIPFC } & 28 & 31 & 69^{\mathrm{a}} \\ \text { L_dmPFC } & 12 & 7 & 81^{\mathrm{a}} \\ \text { L-thalamus } & 15 & 5 & 65^{\mathrm{a}} \\ \text { R_PCC } & 9 & 21 & 77^{\mathrm{a}} \\ \text { IPCC-seed sex } & & & \\ \text { L_insula } & 22 & 84^{\mathrm{a}} & 23 \\ \text { R_mTemporal } & 39 & 55^{\mathrm{a}} & 31 \\ \text { R_sTemporal } & 33 & 72^{\mathrm{a}} & 8 \\ \text { R_insula } & 35 & 85^{\mathrm{a}} & 15\end{array}$

ImPFC/IPCC-seed pubertyXsex

\begin{tabular}{llll} 
L_dmPFC & $67^{\mathrm{a}}$ & 30 & 39 \\
L_Temporal & $71^{\mathrm{a}}$ & 19 & 10 \\
R_dIPFC & $59^{\mathrm{a}}$ & 26 & 30 \\
L_infParietal & $77^{\mathrm{a}}$ & 16 & 5 \\
L_precen & $76^{\mathrm{a}}$ & 34 & 16 \\
R_mTemporal & $81^{\mathrm{a}}$ & 25 & 9 \\
R_dmPFC & $78^{\mathrm{a}}$ & 17 & 28 \\
R_precen & $76^{\mathrm{a}}$ & 38 & 17 \\
\hline
\end{tabular}

Printed values are multiplied by 100 and rounded to the nearest integer. Values $>0.4$ are flagged by an ' $a$ '

Labels: $I=$ left, $r=$ right

pgACC: pregenual anterior cingulate cortex; dLPFC: dorsolateral prefrontal cortex; dmPFC: dorsomedial; PCC: posterior cingulate cortex; mPFC: medial prefrontal cortex; mTemporal: middle temporal cortex; sTemporal: superior temporal cortex; rdmPFC: right dorsomedial prefrontal cortex; precen: precentral cortex

PCC-sex Four clusters loaded on the third factor that was represented by clusters of the IACC seed. All these clusters were modulated by Sex. Factor-3 was thus labeled ACC-Sex.

Three multiple regression analyses were conducted to assess associations between the baseline iFC neural factors and each of the three follow-up (16 yo) behavioral factors.
Table 4 Decomposition of the Puberty by Sex significant effects on iFC

\begin{tabular}{lllll}
\hline Effect of Puberty on iFC & Boys & \multicolumn{3}{l}{ Girls } \\
\hline ImPFC seed & $\boldsymbol{\beta}$ value & $\boldsymbol{P}$ value & $\boldsymbol{\beta}$ value & $\boldsymbol{P}$ value \\
dmPFC & 0.080 & $<0.0005$ & -0.073 & $<0.05$ \\
Middle temporal gyrus & 0.062 & $<0.005$ & -0.084 & $<0.01$ \\
Dorsolateral PFC & 0.038 & $=0.071$ & -0.100 & $<0.005$ \\
IPCC seed & & & & \\
Inferior parietal lobule & 0.074 & $<0.005$ & -0.104 & $<0.005$ \\
Left precentral gyrus & 0.046 & $<0.05$ & -0.108 & $<0.001$ \\
Right Precentral gyrus & 0.040 & $=0.089$ & -0.114 & $<0.001$ \\
dmPFC & 0.075 & $<0.005$ & -0.078 & $<0.05$ \\
Middle temporal gyrus & 0.073 & $<0.005$ & -0.103 & $<0.01$ \\
\hline
\end{tabular}

Correlations between puberty and significant iFC clusters are shown separately for boys and girls, for the ImPFC seed and the IPCC seed. Beta coefficients and $p$ values associated with the simple slopes between pubertal development and iFC are presented separately for boys (middle column) and girls (right column). All models presented in this table control for age and scanner site mPFC: medial prefrontal cortex; dmPFC: dorsomedial prefrontal cortex; PCC: posterior cingulate cortex

\section{Results}

\section{Puberty-X-sex interactions}

All significant puberty-X-sex interactions indicated that pubertal maturation was associated with increasingly weaker connectivity in females, but increasingly stronger connectivity in males. Slopes between puberty and iFC are reported separately for boys and girls in Table 4, and a representative pattern is illustrated in the scatterplot of Fig. 1.

The anterior (lmPFC) and posterior (IPCC) midline cortical seeds revealed a similar pattern of clusters affected by the puberty-X-sex interaction (Table 5). These clusters were within the DMN and between the defaultmode and central-executive networks. The within-DMN clusters included regions in the middle temporal gyrus (BA37) for both seeds, and the inferior parietal lobule (BA40) for the IPCC seed only. The between networks (default-mode with central-executive) clusters were found in the precentral gyrus for both seeds, the frontal eye field (FEF, BA 8) for the IPCC seed, and the dorsolateral PFC (BA 9) for the lmPFC seed (Fig. 2).

The lpgACC-iFC showed no significant clusters in puberty-X-sex analyses.

\section{Sex main effect}

All sex effects followed the same pattern, i.e., stronger iFC in boys than girls.

The iFCs of both the IPCC and lpgACC seeds were modulated by sex, but in quite distinct brain regions (Table 5, Fig. 3). Both seeds showed within-DMN iFC clusters. The within-DMN clusters were found in the 


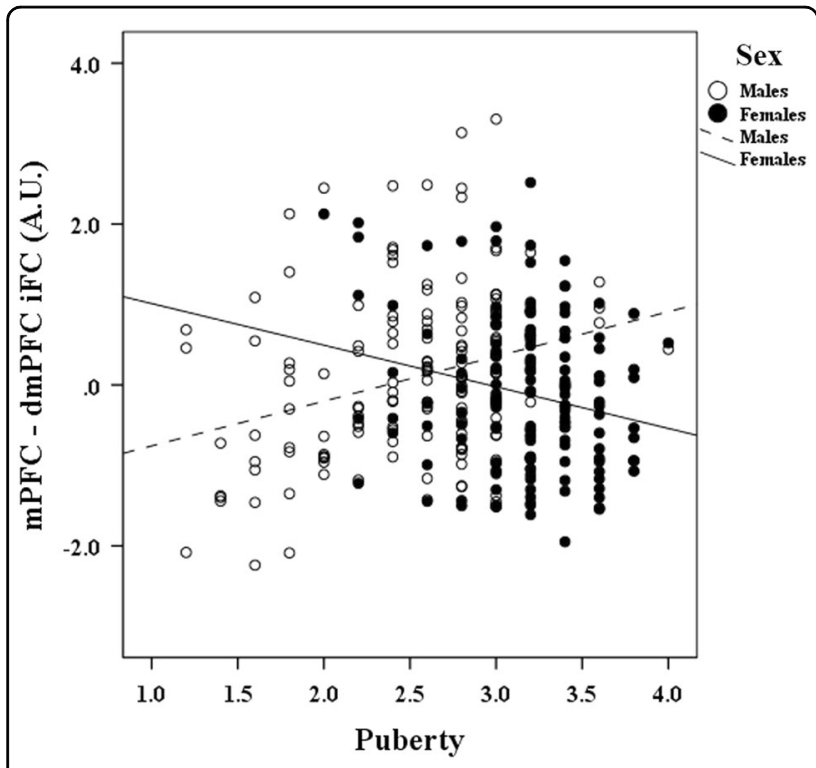

Fig. 1 Scatterplot illustrating a representative relationship between puberty and the iFC of the central-executive network (central-executive network), as a function of sex Specifically, pubertal development ( $x$ axis) is positively associated with the MPFC iFC with dmPFC (y axis) in boys, but negatively associated with the iFC between these regions in girls

right lateral temporal cortex (BA38, BA39) for the IPCC seed, and in the right PCC for the lpgACC seed. Both seeds also showed between-network clusters. For the IPCC, these between-network clusters were located in the right and left insula, which are key nodes of the SN. Regarding the lpgACC, the between-network clusters were found in the left mPFC (BA 10) and left dlPFC (BA9), which are key nodes of the central-executive network. In addition, sex modulated an lpgACC-thalamus (pulvinar) cluster.

Finally, the third seed, the $\operatorname{lmPFC}-\mathrm{iFC}$ revealed a main effect of sex in one cluster located within the right occipital cortex (BA 19) (Table 5).

\section{Puberty main effect}

Other than a main effect of puberty on ImPFC-iFC with the cerebellum $(x=-13.5, y=-79.5, z=-33.5 ; F=$ 20.32; 46 voxels) no clusters were found in cortical or subcortical regions.

\section{Post-hoc analyses}

Based on previous literature, which suggests that small differences in motion during resting-state scans can impact between-group differences in measures of restingstate connectivity ${ }^{91}$, we included average motion per functional volume as a covariate in our analysis of covariance (ANCOVA) models. Controlling for average motion per functional volume did not meaningfully impact the pattern of results reported above (supplemental material, Table S1).

Girls had a significantly higher puberty mean score than boys (Table 1), and post hoc analyses demonstrated that puberty-X-sex interactions were characterized by a positive slope in boys and negative slope in girls, as described above. To ensure that interactions between puberty and sex were not driven by a curvilinear (inverted $U$ ) relationship, where early pubertal maturation was accompanied by increased iFC (more representative of boys) and late pubertal maturation by decreased iFC (more representative of girls), analyses were repeated while controlling for quadratic effects. These analyses relied on power polynomial regression (supplemental material, Table S2). Puberty-X-sex interactions remained significant after controlling for quadratic effects of puberty.

Another way to control for pubertal differences between girls and boys was to match pubertal maturation between sex groups. Boys exhibited lower pubertal development scores than girls (Table 1), and, reciprocally, low levels of pubertal development $(<2)$ were not observed in girls. To ensure that the differential effect of puberty between boys and girls was not driven by non-overlapping developmental stages, we tested for puberty-X-sex interactions within a sample of participants who had pubertal development scores of 2 or greater (supplemental material, Table S3). Importantly, all clusters identified as a function of the interaction within our whole brain analyses remained significant after excluding participants with low PDS scores.

\section{Exploratory brain-behavior analyses}

Multiple regression analyses were conducted to determine whether the three iFC principal components uniquely predicted variance within the three behavioral principal components. The ACC-sex principal component was negatively associated with the principal component characterizing internalizing problems $(\beta=$ $-0.139, p=0.028)$, whereas controlling for other iFC principal components.

\section{Discussion}

To our knowledge, this is the first resting-state fMRI study to examine the effects of puberty and sex, without the confounding effects of chronological age. This study is specifically focused on the resting-state networks that have been previously implicated in youth vulnerability to internalizing problems. The main tenet that drives the present work is that adolescence is a period of huge transformations, particularly at the brain level, and that these changes contribute to the development of psychopathology, such as internalizing disorders. Furthermore, a critical determinant of these changes rests on pubertyrelated action of sex steroids. The uniqueness of this study 
Table 5 Significant iFC of three seeds, left medial PFC, left anterior cingulate cortex, and left posterior cingulate cortex across the whole brain

\begin{tabular}{|c|c|c|c|c|c|c|c|}
\hline & & & \multicolumn{3}{|c|}{ Talaraich } \\
\hline & & Region & Cluster Size (k) & Maxima & $\mathrm{x}$ & $\mathrm{Y}$ & Z \\
\hline & & \multicolumn{6}{|l|}{ Puberty by sex } \\
\hline \multirow[t]{3}{*}{ IMPFC seed } & \multirow[t]{2}{*}{ DMN-CEN } & $L \mathrm{dmPFC}(\mathrm{BA} 6)$ & 223 & 17.16 & -4.5 & 4.5 & 56.5 \\
\hline & & $\mathrm{R}$ dIPFC (BA 9) & 36 & 11.87 & 40.5 & 34.5 & 29.5 \\
\hline & DMN-DMN & $\mathrm{R}$ mTemporal ctx & 41 & 16.10 & 58.5 & -56 & -6.5 \\
\hline \multirow[t]{6}{*}{ IPCC seed } & \multirow[t]{3}{*}{ DMN-CEN } & $\mathrm{R}$ dmPFC (BA 8) & 47 & 16.28 & 7.5 & 25.5 & 44.5 \\
\hline & & L Precentral Ctx (BA 6) & 61 & 15.30 & -47 & 1.5 & 32.5 \\
\hline & & R Precentral Ctx (BA 6) & 70 & 12.28 & 46.5 & 1.5 & 26.5 \\
\hline & \multirow[t]{3}{*}{ DMN-DMN } & R mTemporal Ctx & 68 & 16.42 & 58.5 & -53 & -9.5 \\
\hline & & L Inferior Parietal Ctx & 82 & 24.38 & -44 & -47 & 41.5 \\
\hline & & \multicolumn{6}{|l|}{ Sex } \\
\hline IMPFC seed & & R Occipital Cx & 37 & 17.97 & 31.5 & -89 & 17.5 \\
\hline \multirow[t]{4}{*}{ IPCC seed } & \multirow[t]{2}{*}{$\mathrm{DMN}-\mathrm{SN}$} & L Insula & 41 & 22.34 & -38 & -23 & 11.5 \\
\hline & & R Insula & 89 & 16.55 & 46.5 & -14 & 11.5 \\
\hline & \multirow[t]{2}{*}{ DMN-DMN } & R mTemporal Ctx & 56 & 13.37 & 43.5 & -59 & 14.5 \\
\hline & & R aTemporal Ctx & 47 & 16.42 & 58.5 & 7.5 & -6.5 \\
\hline \multirow[t]{4}{*}{ IpgACC seed } & \multirow[t]{2}{*}{ DMN-CEN } & L mPFC (BA 10) & 142 & 16.60 & -1.5 & 55.5 & 14.5 \\
\hline & & L dIPFC (BA 9) & 102 & 15.57 & -50 & 16.5 & 26.5 \\
\hline & \multirow[t]{2}{*}{ DMN-DMN } & R PCC & 37 & 15.35 & 13.5 & -68 & 14.5 \\
\hline & & L Thalamus & 48 & 17.79 & -7.5 & -23 & 8.5 \\
\hline
\end{tabular}

DMN-DMN reflects within-network iFC, whereas DMN-CEN, and DMN-SN reflect between-network iFC

Of note, the occipital cortex and the thalamus are not associated with specific resting-state networks

is to examine developmental changes within functional brain organization that can be attributed to pubertal maturation, age being held constant, and that these changes are likely to play a role in the vulnerability to internalizing disorders.

Accordingly, the three seeds examined in the present study belong to the $\mathrm{DMN}^{80}$. These seeds have also been shown to be associated with subthreshold elevated ${ }^{40}$, as well as depressed ${ }^{41}$ mood symptoms in two independent subsets of a community cohort of 14 yo (IMAGEN consortium $^{39}$ ). Of note, these cortical regions have also been implicated in the processing of salience and emotion encoding, particularly in the context of social and selfreferential information $(\mathrm{mPFC})^{82}$, visual stimuli $(\mathrm{PCC})^{83}$, and autonomic visceral signals (pgACC) ${ }^{84}$.

Based on the Triple-Network Model ${ }^{55}$ and the focus on vulnerability to emotion dysregulation, three predictions regarding the iFC of the DMN were tested. First, pubertal maturation would impact the DMN iFC differently in girls and boys. Second, sex differences in the DMN iFC would be detected independently of puberty. Third, no sex- independent effects of puberty would be found, given that puberty is by essence sexually dimorphic. Finally, brain-behavior relationships would inform the potential contribution of the DMN to the onset of internalizing problems 2 years later. Findings were broadly in line with predictions.

\section{Puberty-X-sex interaction}

As expected, within- and between-network couplings of the DMN were affected by pubertal maturation differently in girls and boys. These effects were restricted to the lmPFC and IPCC seeds (Table 5). The ImPFC and IPCC connectivity maps revealed similar patterns with regards to iFC topography and sex effects. First, both maps revealed within-network and between-network iFC clusters, the latter specifically with the central-executive network, but not the SN. Second, all findings followed the same motif, i.e., connectivity decreased with puberty in girls, but increased in boys. We speculated that this pattern could be relevant to the emergence of affective dysregulation in adolescence, which affects more girls 


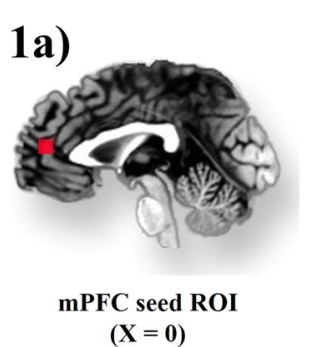

2a)

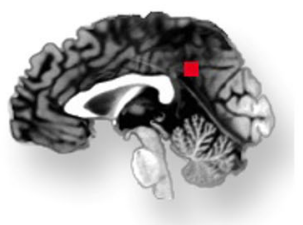

IpCC seed ROI

$(\mathrm{X}=\mathbf{0})$

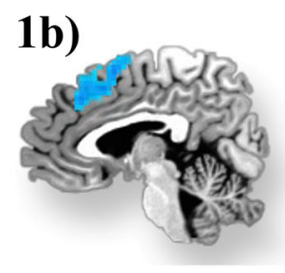

dmPFC

$(\mathrm{X}=\mathbf{4})$

2b)

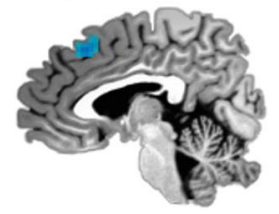

dmPFC

$(\mathrm{X}=\mathbf{4})$

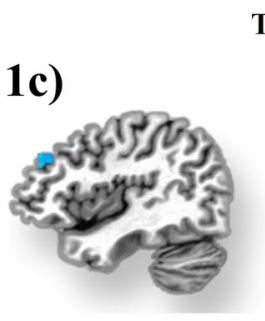

T statistic

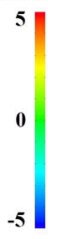

dIPFC

$(X=41)$

2c)

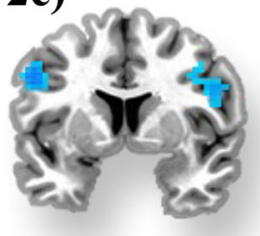

Precentral gyrus

$$
(\mathbf{Y}=\mathbf{2})
$$

Fig. 2 Pubertal development is associated with the iFC of the central-executive network (central-executive network), as a function of sex Upper panel: interactions between puberty and gender characterizing the iFC between the MPFC seed ROI (1a), clusters within the dmPFC (1 b), and dIPFC (1c). Not depicted, is the right middle temporal cortex. Bottom panel: interactions between puberty and sex characterizing the iFC between the pCC seed ROI (2a), the dmPFC (2b), and the bilateral precentral gyrus (2c). Not depicted are the right middle temporal and left inferior parietal cortex

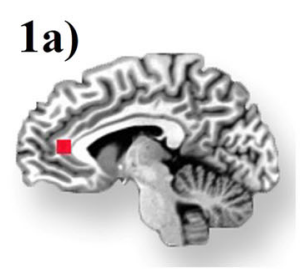

$\operatorname{lpg} \mathrm{ACC}$ seed ROI $(X=-6)$

2a)

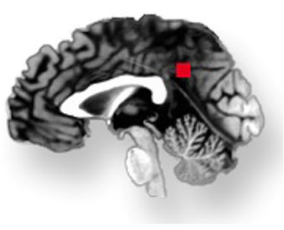

IpCC seed ROI

$$
\mathbf{X}=\mathbf{0}
$$

1b)

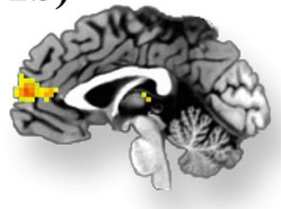

dmPFC

$(X=-1)$

2b)

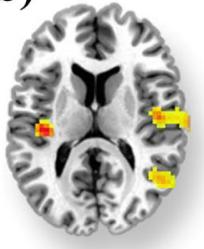

Insula 1c)

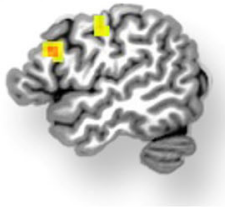

dIPFC

$(X=-49)$

2c)

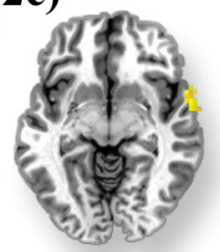

Anterior temporal

cortex

$(Z=-5)$ 1d)

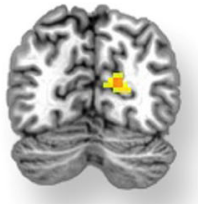

Cuneus

$(\mathrm{Y}=-67)$

2d)

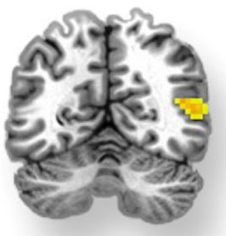

Middle temporal cortex

$(\mathrm{Y}=\mathbf{- 5 8})$ 1e)

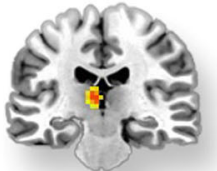

Thalamus

(Y=-22)

Fig. 3 Sex is associated with the iFC of the DMN (default-mode network), the central-executive network (central-executive network), and the salience network (salience network) Upper panel: boys exhibited higher iFC than girls between the IpgACC seed ROI (1a) and clusters within

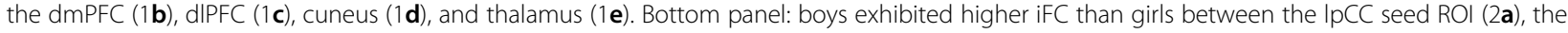
bilateral insula (2b), regions of the anterior $(2 \mathbf{c})$, and middle temporal cortex (2d) 
than boys. Accordingly, the observed sexually dimorphic maturational changes of the DMN might reflect changes in adaptive emotion regulation with puberty. The direction of these changes might be protective in boys, or reflect vulnerability in girls for mood dysregulation.

In support of this thesis, the exploratory brain-behavior analysis across the whole sample revealed a negative association between the spgACC iFC component and internalizing symptoms 2 years later: The lower the connectivity at 14 yo, the more severe were internalizing problems at 16 yo. This relationship suggests that puberty, which is accompanied by decreased iFC in girls, might amplify risk for internalizing symptoms in girls. Findings from the literature using clinical samples or individuals at risk for internalizing disorders could inform this interpretation. Unfortunately, results are inconsistent, in terms of topology, direction of group differences, methodology, and sample characteristics. A number of studies in pediatric samples have reported that internalizing symptoms were associated with lower iFC in core networks. For example, Frost-Bellgowan et al. (2015) ${ }^{92}$ reported lower DMN-iFC (with insula, ventral striatum, pre/post central gyrus) in behavioral inhibited (at risk for internalizing disorders) vs. healthy children ( $8-17$ yo), and showed that girls (not boys) exhibited a negative correlation of internalizing-symptom severity with DMN-iFC. Another study of 8-12-yo children also showed that patients with depression/anxiety (vs. healthy peers) had similarly lower iFC, but this time in the ventral attention network, which was the exclusive focus of this work ${ }^{66}$. In contrast, a relatively large study in a community sample of 112 children ( 53 boys, 59 girls; mean age $=11.5$ yo ${ }^{93}$ focused on the three networks of the triple-network model ${ }^{55}$ in relation to internalizing symptoms (i.e., anxiety and depression, and rumination). Findings revealed no sex differences in the iFCs of the DMN, SN, or CEN. However, brain-behavior correlations identified a positive correlation between iFC within the $\mathrm{SN}$ and the levels of internalizing symptoms in girls, but not in boys. Specifically, higher $\mathrm{SN}$-iFC predicted more severe symptoms in girls. In summary, this study ${ }^{93}$ reported a differential effect of sex on the association of $\mathrm{SN}-\mathrm{iFC}$ with internalizing symptoms, affecting only girls. Compared with our current study, this brain-behavior relationship ${ }^{93}$ concerned a different network (SN) and was in the opposite direction to our findings (greater iFC with higher symptoms, while we showed lower iFC with higher symptoms). Furthermore, it is difficult to compare these results with the present findings, because of the differences in methodology (independent component analysis), and of the significantly younger sample (11yo). Collectively, these pediatric studies concur on the association between dysfunction and internalizing symptoms, but the direction of this dysfunction is inconsistent, as is the specific couplings. In addition, the two studies probing the effect of sex revealed that brain-behavior associations concerned mainly girls. This observation supports our interpretation that the puberty-related reduction in the DMN iFC in girls might carry vulnerability for internalizing symptoms.

Negative findings regarding the distinct iFC modulation by puberty in girls and boys are notable. The lack of sex modulation of the effects of puberty on the lpgACC seed connectivity, and the lack of implication of the SN (particularly the insula) and the amygdala were surprising. Indeed, their established role in the coding of salience and emotional responses (e.g., ref. ${ }^{94,95}$, would be expected to contribute to the increased emotional intensity and lability in adolescence (e.g., ref. ${ }^{60,64,96}$ ). These negative findings suggest that the iFC of the nodes/circuits underlying emotion/motivation processes do mature similarly in boys and girls. Although task-based fMRI studies have reported puberty-related changes of these structures in response to emotion/motivation probes (e.g., ref. ${ }^{20,22,24}$ ), how sex influences these trajectories has not been reported.

\section{Sex main effect}

In fact, the present findings reveal sex effects, independent of puberty, in the spgACC iFC and SN (insula). In all cases, connectivity was higher in boys than girls. These sex effects concerned mainly two of the three seeds, the IPCC and lpgACC.

From the perspective of the Triple-Network Model, sex impacted differently the between-network connectivity of the anterior (spgACC seed) and posterior (IPCC seed) DMN components (Table 5) (anterior vs. posterior hubs of the $\mathrm{DMN}^{97}$ ). Sex influenced the connectivity of the anterior DMN seed (spgACC) with the $\mathrm{SN}$, and the connectivity of the posterior seed (IPCC) with the centralexecutive network. In line with this distinction, as discussed above, the anterior spgACC connectivity predicted internalizing symptoms 2 years later (age 16 years), whereas the posterior IPCC component was not associated with any of the behavioral components.

These differential effects of sex on the anterior and posterior DMN iFC need to be examined more closely in the context of the modulation of the Triple-Network Model, and might suggest a refinement of this model by considering regional functional specialization within the $\mathrm{DMN}$, i.e., the anterior and posterior aspects. Accordingly, they suggest that the modulation by sex and puberty affects differently the anterior and posterior DMN components ${ }^{98}$, which likely play distinct roles in vulnerability to emotion dysregulation.

\section{Limitations}

These findings are not without limitations. First, puberty was measured by self-report $\left(\mathrm{PDS}^{71}\right)$, without 
physical examination or hormonal assays. However, the PDS has been shown to have good psychometric properties and convergent validity from self- and physicianrated Tanner stages ${ }^{71,72}$. Second, as girls and boys were of similar age, the average pubertal level was higher in girls. Two different control analyses were conducted to validate the findings. First, the youngest boys were removed from analysis to equalize the puberty mean in girls and boys. Findings with these control analyses revealed that the pattern of results remained pretty much unchanged (supplemental material, Table S3). Second, we conducted multiple regression analyses, which included quadratic terms for puberty across the whole sample. This approach explored whether the effects of puberty were indeed characterized by two separate and opposite lines of regression in girls and boys, while controlling for an inverse-U-curve pattern of pubertal maturation. Results showed that the puberty-X-sex interaction was still significant (supplemental material, Table S2). Therefore, we are confident in the findings of different pubertal trajectories in boys and girls. Third, this study was cross-sectional, which is sub-optimal for the study of developmental changes. However, this limitation is leveraged by the relatively large sample size, and also the homogeneous age that allowed us to dissociate the effects of chronological changes from those specific to puberty. Fourth, this study focused on extending earlier brain structural findings of neural risk for mood problems in 14 yo. Accordingly, only three seeds were investigated, which constrained the yield of this study. However, this approach is specific to a behavioral domain that, we hope, will foster the formulation of models of vulnerability to internalizing problems to be tested in the future.

\section{Conclusions}

This study reveals that pubertal maturation influences iFC differently in boys and girls, and that sex can impact iFC independently of puberty. First, because mood dysregulation has its peak onset in adolescence, during pubertal maturation, it is reasonable to consider a role of puberty in the rise of incidence rate of mood symptoms. Second, because mood dysregulation, particularly depression and anxiety, occurs disproportionally in girls, sex is expected to uniquely modulate circuits of emotion regulation. For these reasons, analyses were focused on three regions previously identified as conferring risk for mood problems in adolescents. These three regions happened to belong to the DMN, which is recognized to be perturbed in pathological mood disturbances. Findings revealed that both puberty-X-sex interaction and sex main effects modulate within-network and betweennetwork clusters of the DMN iFC. Notably, effects of puberty did not involve the amygdala or the SN, suggesting the notion that pubertal maturation might not significantly affect the iFC of these key centers of emotion processes. This is in contrast to the effect of sex, which did impact DMN-SN iFC. Tentatively, stronger iFC in boys might suggest tighter emotional control, also potentially serving a protective role against emotion dysregulation. Finally, the spgACC iFC significantly predicted internalizing symptoms 2 years later, supporting the association of this network with mood dysregulation.

\section{Acknowledgements}

Dr Stringaris receives royalties from the University of Cambridge for his book. Dr. Banaschewski served in an advisory or consultancy role for Actelion, Hexal Pharma, Lilly, Medice, Novartis, Oxford outcomes, PCM scientific, Shire, and Viforpharma. He received conference support or speaker's fee by Medice, Novartis, and Shire. He is/has been involved in clinical trials conducted by Shire \& Viforpharma. He received royalities from Hogrefe, Kohlhammer, CIP Medien, Oxford University Press. The present work is unrelated to the above grants and relationships. This work received support from the following funding sources: the European Union-funded FP6 Integrated Project IMAGEN (Reinforcementrelated behavior in normal brain function and psychopathology) (LSHM-CT2007-037286), the Agence Nationale de la Recherche ANR (project AF12NEUR0008-01-WM2NA, and ANR-12-SAMA-0004), the Fondation de France (grants 2012-00033703 and 2017-0008L242), the Fondation pour la Recherche Médicale (grants DPA 20140229802 and DPP20151033945), the Fédération pour la Recherche sur le Cerveau (AAP 2014), the Mission Interministérielle de Lutte-contre-les-Drogues-et-les-Conduites-Addictives (MILDECA), and the INSERM (interface grant to the the Assistance-PubliqueHôpitaux-de-Paris); The Academy of Finland (grant number 276612 to A.S.U); the Emil Aaltonen Foundation (grant to A.S.U.); the Jalmari and Rauha Ahokas Foundation (grant to A.S.U.). Further support to the IMAGEN centers in UK and Germany was provided by the Horizon 2020 funded ERC Advanced Grant 'STRATIFY' (Brain network based stratification of reinforcement-related disorders) (695313), ERANID (Understanding the Interplay between Cultural, Biological, and Subjective Factors in Drug Use Pathways) (PR-ST-0416-10004), BRIDGET (JPND: BRain Imaging, cognition Dementia and next generation GEnomics) (MR/N027558/1), the FP7 projects IMAGEMEND(602450; IMAging GEnetics for MENtal Disorders), and MATRICS (603016), the Innovative Medicine Initiative Project EU-AIMS (115300-2), the Medical Research Council Grant 'C-VEDA' (Consortium on Vulnerability to Externalizing Disorders and Addictions) (MR/N000390/1), the Swedish Research Council FORMAS, the Medical Research Council, the National Institute for Health Research (NIHR) Biomedical Research Centre at South London and Maudsley NHS Foundation Trust and King's College London, the Bundesministeriumfür Bildung und Forschung (BMBF grants 01GS08152; 01EV0711; eMED SysAlc01ZX1311A; Forschungsnetz AERIAL 01EE1406A, 01EE1406B), the Deutsche Forschungsgemeinschaft (DFG grants SM 80/7-2, SFB 940/2), the Medical Research Foundation and Medical research council (grant MR/R00465X/1). Further support was provided by grants from: the National Institutes of Health, Science Foundation Ireland (16/ERCD/3797), USA (Axon, Testosterone, and Mental Health during Adolescence; RO1 MH085772-01A1), and by NIH Consortium grant U54 EB020403, supported by a cross-NIH alliance that funds Big Data to Knowledge Centres of Excellence. This work was supported by the European Union-funded FP6 Integrated Project IMAGEN (LSHM-CT-2007037286); the FP7 project IMAGEMEND (602450); the Innovative Medicine Initiative Project European Autism Interventions-A Multicentre Study for Developing New Medications (EU-AIMS) (115300-2); a Medical Research Council Programme (93558); the Swedish funding agency Formas; the Wellcome Trust (University of Cambridge); the National Institute for Health Research (NIHR) Biomedical Research Centre at South London and Maudsley NHS Foundation Trust and King's College London; the Department of Health United Kingdom; the Bundesministerium für Bildung und Forschung (BMBF) (01GS08152, 01EV0711, eMED SysAlc01ZX1311A, Forschungsnetz AERIAL); the French funding agency Agence Nationale de la Recherche (ANR) (ANR-12SAMA-0004); Eranet-Neuron (AF12-NEUR0008-01-WM2NA); the AssistancePublique-Hôpitaux-de-Paris and Insitut national de la santé et de la recherché médicale (INSERM, interface grant); Paris-Descartes-University (collaborativeproject-2010); Paris-Sud-University (IDEX-2012); the Fondation de France; the Fondation pour la Recherche Médicale (DPA20140629802); the Mission Interministérielle de Lutte-contre-les-Drogues-et-les-Conduites-Addictives 
(MILDECA); and the Intramural Research Program of the National Institute of Mental Health (ZIAMH002798).

\section{Other Imagen Consortium collaborators (www.imagen-europe.com):}

J. Dalley, N. Subramaniam, D. Theobald, C. Bach, G. J. Barker, M. Fauth-Bühler, S Millenet, R. Spanagel, L. Albrecht, N. Ivanov, M. Rapp, J. Reuter, N. Strache, A. Ströhle, J. B. Poline, Y. Schwartz, B. Thyreau, J. Ireland, J. Rogers, N. Bordas, Z. Bricaud, I. Filippi, A. Galinowski, F. Gollier-Briant, D. Hall, S. Havatzias, T. Jia, C. Mallik, C. Nymberg, B. Ruggeri, L. Smith, K. Stueber, L. Topper, H. Werts, R. Brühl R. A. Ihlenfeld, B. Walaszek, T. Hübner, K. Müller, T. Paus, S. Ripke, E. Mennigen, D. Schmidt, N. C. Vetter, V. Ziesch, D. Carter, C. Connolly, S. Nugent, J. Jones, J. Yacubian, S. Schneider, K. Head, N. Heym, C. Newman, Z. Pausova, A. Tahmasebi, D. Stephens.

\section{Author details}

${ }^{1} \mathrm{NIMH} / \mathrm{NIH}$, Bethesda, MD, USA. ${ }^{2}$ INSERM, UMR 1000, Research unit "Neuroimaging and Psychiatry", DIGITEO Labs, University Paris-Saclay, and University Paris Descartes, Gif sur Yvette, France. ${ }^{3}$ INSERM, UMR 1000, Faculté de médecine, University Paris-Saclay, DIGITEO Labs, Gif sur Yvette, France. ${ }^{4}$ University Paris Descartes, Paris, France. ${ }^{5}$ Center for Neuroimaging Research (CENIR), Brain \& Spine Institute, Paris, France. 'Psychiatry Department 91G16, Orsay Hospital, Paris, France. ${ }^{7}$ Department of Child and Adolescent Psychiatry and Psychotherapy, Central Institute of Mental Health, Medical Faculty Mannheim, Heidelberg University, Mannheim, Germany. ${ }^{8}$ Discipline of Psychiatry, School of Medicine and Trinity College Institute of Neurosciences, Trinity College, Dublin, Ireland. ' University Medical Centre HamburgEppendorf, House W34, 3.OG, Hamburg, Germany. ${ }^{10}$ Physikalisch-Technische Bundesanstalt, Abbestr. 2 - 12, Berlin, Germany. ${ }^{11}$ Medical Research Council Social, Genetic and Developmental Psychiatry Centre, Institute of Psychiatry, Psychology \& Neuroscience, King's College London, London, United Kingdom. ${ }^{12}$ Department of Psychological Medicine and Psychiatry, Institute of Psychiatry, Psychology \& Neuroscience, King's College London, London, United Kingdom. ${ }^{13}$ Department of Psychiatry, Université de Montréal, CHU Ste Justine Hospital, Montréal, QC, Canada. ${ }^{14}$ Department of Psychology, School of Social Sciences, University of Mannheim, 68131 Mannheim, Germany. ${ }^{15}$ Neurospin, Commissariat à l'Energie Atomique, CEA-Saclay Center, Saclay, France. ${ }^{16}$ Department of Psychiatry and Psychotherapy, University Medical Center Hamburg-Eppendorf, Martinistr. 52, 20246 Hamburg, Germany. ${ }^{17}$ Departments of Psychiatry and Psychology, University of Vermont, 05405 Burlington, VT, USA. ${ }^{18}$ Sir Peter Mansfield Imaging Centre School of Physics and Astronomy, University of Nottingham, University Park, Nottingham, United Kingdom. ${ }^{19}$ Department of Psychiatry and Psychotherapy, Campus CharitéMitte, CharitéUniversitätsmedizin Berlin, Charitéplatz 1, Berlin, Germany. ${ }^{20}$ Department of Child and Adolescent Psychiatry Psychosomatics and Psychotherapy, Campus CharitéMitte, Charité-Universitätsmedizin Berlin, Charitéplatz 1, Berlin, Germany.

${ }^{21}$ Department of Social and Health Care, Psychosocial Services Adolescent Outpatient Clinic, University of Tampere, Kauppakatu 14, Lahti, Finland. ${ }^{22}$ Department of Child and Adolescent Psychiatry and Psychotherapy, Medical University of Vienna, Vienna, Austria. ${ }^{23}$ Department of Psychiatry and Neuroimaging Center, Technische Universität Dresden, Dresden, Germany. ${ }^{24}$ Department of Child and Adolescent Psychiatry, Institute of Psychiatry, Psychology \& Neuroscience, King's College London, London, United Kingdom. ${ }^{25}$ Department of Psychology, University College, Dublin, Ireland. ${ }^{26}$ AP-HP, Department of Child and Adolescent Psychiatry, Pitié-Salpêtrière Hospital, Paris, France. ${ }^{27}$ Sorbonne Universités, Paris, France

\section{Conflict of interest}

All the other authors declare no biomedical financial interests or potential conflict of interest.

\section{Publisher's note}

Springer Nature remains neutral with regard to jurisdictional claims in published maps and institutional affiliations.

Supplementary information accompanies this paper at (https://doi.org/ 10.1038/s41398-019-0433-6).
Received: 3 May 2018 Revised: 11 October 2018 Accepted: 1 January 2019 Published online: 25 February 2019

\section{References}

1. Blakemore, S. J., Burnett, S. \& Dahl, R. E. The role of puberty in the developing adolescent brain. Hum. Brain Mapp. 31, 926-933 (2010).

2. Bale, T. L. \& Epperson, C. N. Sex as a biological variable: who, what, when, why and How. Neuropsychopharmacology 42, 386-396 (2017).

3. Forbes, E. E. \& Dahl, R. E. Pubertal development and behavior: Hormonal activation of social and motivational tendencies. Brain Cogn. 72, 66-72 (2010).

4. Byrne, M. L. et al. A systematic review of adrenarche as a sensitive period in neurobiological development and mental health. Dev. Cogn. Neurosci. 25, 12-28 (2017).

5. Kessler, R. C. et al. Lifetime prevalence and age-of-onset distributions of DSMIV disorders in the National Comorbidity Survey Replication. Arch. Gen. Psychiatry 62, 593-602 (2005).

6. Kessler, R. C., Petukhova, M., Sampson, N. A., Zaslavsky, A. M. \& Wittchen, H. U. Twelve-month and lifetime prevalence and lifetime morbid risk of anxiety and mood disorders in the United States. Int J. Methods Psychiatr. Res. 21, 169-184 (2012).

7. Whittle, S. et al. Associations between early adrenarche, affective brain function and mental health in children. Soc. Cogn. Affect Neurosci. 10, 1282-1290 (2015).

8. Casey, B. J., Soliman, F., Bath, K. G. \& Glatt, C. E. Imaging genetics and development: challenges and promises. Hum. Brain Mapp. 31, 838-851 (2010).

9. Casey, B. J., Duhoux, S. \& Malter Cohen, M. Adolescence: what do transmission, transition, and translation have to do with it? Neuron 67, 749-760 (2010).

10. Giedd, J. N. et al. Development of the human corpus callosum during childhood and adolescence: a longitudinal MRI study. Progress euroPsychopharmacol. Biol. Psychiatry 23, 571-588 (1999).

11. Matsuzawa, J. et al. Age-related volumetric changes of brain gray and white matter in healthy infants and children. Cereb. Cortex 11, 335-342 (2001).

12. Shaw, P. et al. Neurodevelopmental trajectories of the human cerebral cortex. J. Neurosci. 28, 3586-3594 (2008).

13. Jung, R. E. \& Haier, R. J. The Parieto-Frontal Integration Theory (P-FIT) of intelligence: converging neuroimaging evidence. Behav. rain Sci. 30, 135-154 (2007). discussion154-187.

14. Deary, I. J., Penke, L. \& Johnson, W. The neuroscience of human intelligence differences. Nat. Rev. Neurosci. 11, 201-211 (2010).

15. De Bellis, M. D. et al. Sex differences in brain maturation during childhood and adolescence. Cereb. Cortex 11, 552-557 (2001).

16. Goldstein, J. M. et al. Normal sexual dimorphism of the adult human brain assessed by in vivo magnetic resonance imaging. Cereb. Cortex 11, 490-497 (2001).

17. Lenroot, R. K. \& Giedd, J. N. Sex differences in the adolescent brain. Brain Cogn. 72, 46-55 (2010).

18. Nota, N. M. et al. Brain functional connectivity patterns in children and adolescents with gender dysphoria: Sex-atypical or not? Psychoneuroendocrinology 86, 187-195 (2017).

19. Giedd, J. N. et al. Puberty-related influences on brain development. Mol. Cell Endocrinol. 254-255, 154-162 (2006).

20. Forbes, E. E., Phillips, M. L., Silk, J. S., Ryan, N. D. \& Dahl, R. E. Neural systems of threat processing in adolescents: role of pubertal maturation and relation to measures of negative affect. Dev. Neuropsychol. 36, 429-452 (2011).

21. Klapwijk, E. T. et al. Increased functional connectivity with puberty in the mentalising network involved in social emotion processing. Horm. Behav. 64, 314-322 (2013).

22. Moore, W. E. 3rd et al. Facing puberty: associations between pubertal development and neural responses to affective facial displays. Soc. Cogn. Affect Neurosci. 7, 35-43 (2012).

23. Alarcon, G., Cservenka, A., Fair, D. A. \& Nagel, B. J. Sex differences in the neura substrates of spatial working memory during adolescence are not mediated by endogenous testosterone. Brain Res. 1593, 40-54 (2014).

24. Goddings, A. L., Burnett Heyes, S., Bird, G., Viner, R. M. \& Blakemore, S. J. The relationship between puberty and social emotion processing. Dev. Sci. 15 801-811 (2012)

25. Satterthwaite, T. D. et al. Impact of puberty on the evolution of cerebral perfusion during adolescence. Proc. Natl Acad. Sci. USA 111, 8643-8648 (2014). 
26. Cservenka, A., Stroup, M. L., Etkin, A. \& Nagel, B. J. The effects of age, sex, and hormones on emotional conflict-related brain response during adolescence. Brain Cogn. 99, 135-150 (2015).

27. Vijayakumar, N., Op de Macks, Z., Shirtcliff, E. A. \& Pfeifer, J. H. Puberty and the human brain: Insights into adolescent development. Neurosci. Biobehav Rev. 92, 417-436 (2018).

28. Satterthwaite, T. D. et al. Neuroimaging of the Philadelphia neurodevelopmental cohort. Neuroimage 86, 544-553 (2014).

29. Dennison, $\mathbf{M}$. et al. Mapping subcortical brain maturation during adolescence: evidence of hemisphere- and sex-specific longitudinal changes. Dev. Sci. 16, 772-791 (2013)

30. Vijayakumar, $\mathrm{N}$. et al. Brain development during adolescence: a mixedlongitudinal investigation of cortical thickness, surface area, and volume. Hum. Brain Mapp. 37, 2027-2038 (2016).

31. Wierenga, L. M. et al. Unraveling age, puberty and testosterone effects on subcortical brain development across adolescence. Psychoneuroendocrinology 91, 105-114 (2018).

32. Ernst, M., Torrisi, S., Balderston, N., Grillon, C. \& Hale, E. A. fMRl functional connectivity applied to adolescent neurodevelopment. Annu. Rev. Clin. Psychol. 11, 361-377 (2015)

33. Satterthwaite, T. D. et al. Linked sex differences in cognition and functional connectivity in youth. Cereb. cortex 25, 2383-2394 (2015).

34. Philippi, C. L., Motzkin, J. C., Pujara, M. S. \& Koenigs, M. Subclinical depression severity is associated with distinct patterns of functional connectivity for subregions of anterior cingulate cortex. J. Psychiatr. Res. 71, 103-111 (2015).

35. Weissman-Fogel, I., Moayedi, M., Taylor, K. S., Pope, G. \& Davis, K. D. Cognitive and default-mode resting state networks: do male and female brains "rest" differently? Hum. Brain Mapp. 31, 1713-1726 (2010).

36. Zhang, C. et al. Sex and age effects of functional connectivity in early adulthood. Brain Connect. 6, 700-713 (2016).

37. Zhang, C., Dougherty, C. C., Baum, S. A., White, T. \& Michael, A. M. Functional connectivity predicts gender: evidence for gender differences in resting brain connectivity. Hum. Brain Mapp. 39, 1765-1776 (2018).

38. Sato, J. R. et al. Default mode network maturation and psychopathology in children and adolescents. J. Child Psychol. Psychiatry 57, 55-64 (2016).

39. Schumann, G. et al. The IMAGEN study: reinforcement-related behaviour in normal brain function and psychopathology. Mol. Psychiatry 15, 1128-1139 (2010).

40. Paillère Martinot, M. L. et al. White-matter microstructure and gray-matter volumes in adolescents with subthreshold bipolar symptoms. Mol. Psychiatry 19, 462-470 (2014).

41. Vulser, $\mathrm{H}$. et al. Subthreshold depression and regional brain volumes in young community adolescents. J. Am. Acad. Child Adolesc. Psychiatry 54, 832-840 (2015).

42. Raichle, M. E. The brain's default mode network. Annu. Rev. Neurosci. 38, 433-447 (2015).

43. Greicius, M. D., Krasnow, B., Reiss, A. L. \& Menon, V. Functional connectivity in the resting brain: a network analysis of the default mode hypothesis. Proc. Natl Acad. Sci. USA 100, 253-258 (2003).

44. Whitfield-Gabrieli, S. \& Ford, J. M. Default mode network activity and connectivity in psychopathology. Annu Rev. Clin. Psychol. 8, 49-76 (2012).

45. Lichenstein, S. D., Verstynen, T. \& Forbes, E. E. Adolescent brain development and depression: a case for the importance of connectivity of the anterior cingulate cortex. Neurosci. Biobehav. Rev. 70, 271-287 (2016).

46. Yang, R. et al. Decreased functional connectivity to posterior cingulate cortex in major depressive disorder. Psychiatry Res.: Neuroimaging. 255, 15-23 (2016).

47. Raichle, M. E. et al. A default mode of brain function. Proc. Natl Acad. Sci. 98 , 676-682 (2001)

48. Buckner, R. L., Andrews-Hanna, J. R. \& Schacter, D. L. The brain's default network: anatomy, function, and relevance to disease. Ann. NY Acad. Sci. 1124, 1-38 (2008)

49. Rive, M. M. et al. Neural correlates of dysfunctional emotion regulation in major depressive disorder. A systematic review of neuroimaging studies. Neurosci. Biobehav. Rev. 37, 2529-2553 (2013).

50. Pfeifer, J. H. \& Peake, S. J. Self-development: Integrating cognitive, socioemotional, and neuroimaging perspectives. Dev. Cogn. Neurosci. 2, 55-69 (2012).

51. Pizzagalli, D. A. Frontocingulate dysfunction in depression: toward biomarkers of treatment response. Neuropsychopharmacology 36, 183-206 (2011).

52. Qin, P. \& Northoff, G. How is our self related to midline regions and the default-mode network? Neuroimage 57, 1221-1233 (2011).
53. Nejad, A. B., Fossati, P. \& Lemogne, C. Self-referential processing, rumination, and cortical midline structures in major depression. Front. Hum. Neurosci. 7, 666 (2013).

54. Lichenstein, S. D., Verstynen, T. \& Forbes, E. E. Adolescent brain development and depression: a case for the importance of connectivity of the anterior cingulate cortex. Neurosci. Biobehav Rev. 70, 271-287 (2016).

55. Menon, V. Large-scale brain networks and psychopathology: a unifying triple network model. Trends Cogn. Sci. 15, 483-506 (2011).

56. Corbetta, M. \& Shulman, G. L. Control of goal-directed and stimulus-driven attention in the brain. Nat. Rev. Neurosci. 3, 201-215 (2002).

57. Damoiseaux, J. S. S. et al. Consistent resting-state networks across healthy subjects. Proc. Natl Acad. Sci. USA 103, 13848-13853 (2006).

58. Rogers T. T., McClelland J. L. J. Semantic cognition: a parallel distributed processing approach. Cambridge, MA: MIT Press (2013).

59. Sridharan, D., Levitin, D. J. D. J. \& Menon, V. A critical role for the right frontoinsular cortex in switching between central-executive and default-mode networks. Proc. Natl Acad. Sci. 105, 12569-12574 (2008).

60. Ernst, M. The triadic model perspective for the study of adolescent motivated behavior. Brain Cogn. 89, 104-111 (2014).

61. Shulman, E. P. et al. The dual systems model: Review, reappraisal, and reaffirmation. Dev. Cogn. Neurosci. 17, 103-117 (2016).

62. Blakemore, S. J. The social brain in adolescence. Nat. Rev. Neurosci. 9, 267-277 (2008).

63. Galvan, A. Neural systems underlying reward and approach behaviors in childhood and adolescence. Curr. Top. Behav. Neurosci. 16, 167-188 (2014).

64. Nelson, E. E, Leibenluft, E, McClure, E. B. \& Pine, D. S. The social re-orientation of adolescence: a neuroscience perspective on the process and its relation to psychopathology. Psychol. Med. 35, 163-174 (2005).

65. Frost Bellgowan, J. et al. A neural substrate for behavioral inhibition in the risk for major depressive disorder. J. Am. Acad. Child Adolesc. Psychiatry 54, 841-848 (2015)

66. Sylvester, C. M. et al. Resting state functional connectivity of the ventral attention network in children with a history of depression or anxiety. J. Am. Acad. Child Adolesc. Psychiatry 52, 1326-1336.e1325 (2013).

67. Gabard-Durnam, L. J. et al. The development of human amygdala functional connectivity at rest from 4 to 23 years: a cross-sectional study. Neuroimage $\mathbf{9 5}$, 193-207 (2014).

68. Axelrod, B. N. Validity of the Wechsler abbreviated scale of intelligence and other very short forms of estimating intellectual functioning. Assessment $\mathbf{9}$, 17-23 (2002).

69. Goodman, R., Ford, T., Richards, H., Gatward, R. \& Meltzer, H. The development and well-being assessment: description and initial validation of an integrated assessment of child and adolescent psychopathology. J. Child Psychol. Psychiatry. 41, 645-655 (2000)

70. Goodman, R. The Strengths and Difficulties Questionnaire: a research note. J. Child Psychol. Psychiatry 38, 581-586 (1997).

71. Petersen, A. C., Crockett, L., Richards, M. \& Boxer, A. A self-report measure of pubertal status: reliability, validity, and initial norms. J. Youth Adolesc. 17, 117-133 (1988)

72. Siegel, J. M., Yancey, A. K., Aneshensel, C. S. \& Schuler, R. Body image, perceived pubertal timing, and adolescent mental health. J. Adolesc. Health 25, 155-165 (1999).

73. Cox, R. W. AFNl: software for analysis and visualization of functional magnetic resonance neuroimages. Comput. Biomed. Res. 29, 162-173 (1996).

74. Fischl, B. FreeSurfer. Neurolmage 62, 774-781 (2012).

75. Talairach J., Tournoux P. Co-planar Stereotaxic Atlas of the Human Brain. New York: Thieme (1988)

76. Saad, Z. S. et al. Trouble at rest: how correlation patterns and group differences become distorted after global signal regression. Brain Connect. 2, 25-32 (2012).

77. Power, J. D., Plitt, M., Laumann, T. O. \& Martin, A. Sources and implications of whole-brain fMRI signals in humans. Neuroimage 146, 609-625 (2017).

78. Jo, H. J., Saad, Z. S., Simmons, W. K., Milbury, L. A. \& Cox, R. W. Mapping sources of correlation in resting state FMRI, with artifact detection and removal. Neurolmage 52, 571-582 (2010).

79. Power, J. D. et al. Methods to detect, characterize, and remove motion artifact in resting state fMRI. Neuroimage 84, 320-341 (2014).

80. Broyd, S. J. et al. Default-mode brain dysfunction in mental disorders: a systematic review. Biobehav Rev. 33, 279-296 (2009).

81. Buckner, R. L. et al. Cortical hubs revealed by intrinsic functional connectivity: mapping, assessment of stability, and relation to Alzheimer's disease. J. Neurosci. 29, 1860-1873 (2009). 
82. Davey, C. G., Pujol, J. \& Harrison, B. J. Mapping the self in the brain's default mode network. Neurolmage 132, 390-397 (2016).

83. Maddock, R. J., Garrett, A. S. \& Buonocore, M. H. Posterior cingulate cortex activation by emotional words: $\mathrm{fMRl}$ evidence from a valence decision task. Human. Brain Mapp. 18, 30-41 (2003).

84. Vogt, B. A. Pain and emotion interactions in subregions of the cingulate gyrus. Nat. Rev. Neurosci. 6, 533-544 (2005).

85. Chen, G., Adleman, N. E., Saad, Z. S., Leibenluft, E. \& Cox, R. W. Applications of multivariate modeling to neuroimaging group analysis: a comprehensive alternative to univariate general linear model. Neurolmage 99, 571-588 (2014).

86. Eklund A., Nichols T. E., Knutsson H. Cluster failure: why fMRI inferences for spatial extent have inflated false-positive rates. Proc. Natl Acad. Sci. 111:7900-7905 (2016)

87. Cox R. W., Reynolds R. C., Taylor P. A. AFNI and Clustering: false positive Rates Redux (2016)

88. Hayes A. F. PROCESS: A versatile computational tool for observed variable mediation, moderation, and conditional process modeling (2012).

89. Watson, D. Rethinking the mood and anxiety disorders: a quantitative hierarchical model for DSM-V. J. Abnorm. Psychol. 114, 522-536 (2005).

90. Sunderland, M., Mewton, L., Slade, T. \& Baillie, A. J. Investigating differential symptom profiles in major depressive episode with and without generalized anxiety disorder: true co-morbidity or symptom similarity? Psychol. Med. $\mathbf{4 0}$ 1113-1123 (2010)
91. van Dijk, K. R. A., Sabuncu, M. R. \& Buckner, R. L. The influence of head motion on intrinsic functional connectivity MRI. Neurolmage 59, 431-438 (2012).

92. Frost Bellgowan, J. et al. A neural substrate for behavioral inhibition in the risk for major depressive disorder. J Am Acad. Adolesc. Psychiatry 54, 841-848 (2015).

93. Ordaz, S. J. et al. Ruminative brooding is associated with salience network coherence in early pubertal youth. Soc. Cogn. Affect Neurosci. 12, 298-310 (2017).

94. Burghy, C. A. et al. Developmental pathways to amygdala-prefrontal function and internalizing symptoms in adolescence. Nat. Neurosci. 15, 1736-1741 (2012).

95. Pessoa, L. A network model of the emotional brain. Trends Cogn. Sci. 21 357-371 (2017).

96. Luciana, M. Commentary on the special issue on the adolescent Brain: incentive-based striving and the adolescent brain. Neurosci. Biobehav Rev. 70, 339-342 (2016).

97. Andrews-Hanna, J. R. J. R., Reidler, J. S. J. S., Sepulcre, J., Poulin, R. \& Buckner, R. L. R. L. Functional-anatomic fractionation of the brain's default network. Neuron 65, 550-562 (2010).

98. Uddin, L. Q., Kelly, A. M. C., Biswal, B. B., Castellanos, F. X. \& Milham, M. P. Functional connectivity of default mode network components: correlation, anticorrelation, and causality. Hum. Brain Mapp. 30, 1-25 (2009). 